Tropical Journal of Pharmaceutical Research January 2019; 18 (1): 109-115

ISSN: $1596-5996$ (print); 1596-9827 (electronic)

(c) Pharmacotherapy Group, Faculty of Pharmacy, University of Benin, Benin City, 300001 Nigeria.

\title{
Antiurolithiatic effect of ferulic acid on ethylene glycol- induced renal calculus in experimental rats
}

\author{
Bo Zhao ${ }^{1}$, Baozhu Su${ }^{1}$, Hui Zhang ${ }^{2}$, Wei Liu ${ }^{2}$, Qiao $\mathrm{Du}^{2}$, Yingyi Li ${ }^{1 *}$ \\ ${ }^{1}$ Department of Urology, ${ }^{2}$ Department of Medical Imaging, Baoji People's Hospital, Baoji, Shaanxi 721000, People's Republic of \\ China
}

*For correspondence: Email: liuw0303bj@sina.com; Tel: +86-13379478181

Sent for review: 14 August 2018

Revised accepted: 24 December 2018

\begin{abstract}
Purpose: To evaluate the anti-urolithiatic effect of ferulic acid on ethylene glycol-induced kidney stone in a rat model.

Methods: Five groups of adult male Sprague-Dawley rats (6 rats/group) were used in this study. Group I rats served as normal control. Renal calculus was induced through ethylene glycol $(0.75 \% \mathrm{~V} / \mathrm{V}$ in drinking water) administration to all rats for 28 days except those in group 1. Prior to ethylene glycol treatment, ferulic acid was given orally (gavage) to rats in groups III and IV at doses of 40 and 80 $\mathrm{mg} / \mathrm{kg}$, respectively. Rats in group $V$ (positive control) were treated with standard drug, cystone at a dose of $750 \mathrm{mg} / \mathrm{kg}$ prior to ethylene glycol administration, while group II rats received no treatment. Kidney tissue and blood serum were sampled after 28 days and used for biochemical and histopathological analyses.

Results: Rats in group II showed significant increases in oxidative stress pattern as seen in significant reductions in GSH, SOD, GPX, and CAT levels, and significant elevation of lipid peroxidation (LPO), relative to normal control group $(p<0.05)$. However, renal calculus formation and oxidative stress were significantly inhibited by ferulic acid treatment in groups III and IV. These results were supported by histopathological findings.

Conclusion: Ferulic acid exerts anti-urolithiatic effect via inhibition of oxidative stress. Thus, it has potentials for use in preventing kidney stone formation.
\end{abstract}

Keywords: Urolithiasis, Ferulic acid, Ethylene glycol, Kidney, Histopathology

This is an Open Access article that uses a funding model which does not charge readers or their institutions for access and distributed under the terms of the Creative Commons Attribution License (http://creativecommons.org/licenses/by/4.0) and the Budapest Open Access Initiative (http://www.budapestopenaccessinitiative.org/read), which permit unrestricted use, distribution, and reproduction in any medium, provided the original work is properly credited.

Tropical Journal of Pharmaceutical Research is indexed by Science Citation Index (SciSearch), Scopus, International Pharmaceutical Abstract, Chemical Abstracts, Embase, Index Copernicus, EBSCO, African Index Medicus, JournalSeek, Journal Citation Reports/Science Edition, Directory of Open Access Journals (DOAJ), African Journal Online, Bioline International, Open-J-Gate and Pharmacy Abstracts

\section{INTRODUCTION}

Urolithiasis or renal calculi formation has become a prevailing urological complaint among adults all over the world [1]. Bio-mineralization in the kidney, predominantly formation of calcium oxalate is considered a risk factor for urolithiasis. Surgical methods and endoscopy are not completely reliable as treatment for urolithiasis since most of the cases have shown recurrence of renal calculi within a period of 10 years or more [2]. Due to the high cost for surgical treatment and the risk of recurrence of kidney stones, an alternative treatment strategy has become necessary. Untreated renal calculi will eventually lead to renal dysfunction and detrimental health conditions [3]. 
Medicinal plant extracts with strong antioxidant potential have shown anti-urolithiatic effects [4]. Understanding the pathophysiology of renal calculi formation and the effect of antioxidants against the process have resulted in the idea of using phyto-compounds as potential therapy for the disease [5]. Oxidative injury caused by the lithogenic effect of ethylene glycol is known to induce formation of renal calculi in rat models, because epithelial cell damage is a crucial factor in kidney stone formation [6]. Therefore, ethylene glycol-induced renal calculi formation in rats is a popular in vivo model for anti-urolithiasis studies. Reactive oxygen species (ROS) are fundamental mediators of vast biological processes. These free radicals are harmful during oxidative stress, causing injuries which lead to serious ailments [7]. Antioxidants neutralize the damaging effects of free radicals on tissues by donating electrons at the site of injury [8]. Natural antioxidant supplementation is the best option for overcoming oxidative stress if the physiological antioxidant defenses are overwhelmed by overproduction of ROS [9]. Therefore, naturally occurring antioxidants found mostly in plants are used for experimental and therapeutic purposes [10]. The pharmacological effects of natural antioxidants have been documented through numerous studies involving in vitro and in vivo models [11].

In this study, antiurolithic potential of ferulic acid was evaluated against ethylene glycol induced renal calculi formation in rats. . Ferulic acid is a phenolic compound which is largely found on the cell walls of vegetables and fruits [12]. In traditional Chinese medicine, ferulic acid containing plants such as Angelica sinensis, Ligusticum chuangxiong, and Cimicifuga heracleifolia are used for the treatment of several diseases. Studies on the pharmacological properties of ferulic have revealed that it possesses anti-cancer, hepatoprotective, antiinflammatory, anti-diabetic, anti-aging, neuroprotective, anti-atherogenic, and radioprotective effects [13]. The pharmacological properties of ferulic acid are attributed to its strong antioxidant activity. The present study is the first investigation on the in vivo antiurolithiatic properties of ferulic acid.

\section{EXPERIMENTAL}

\section{Materials}

Ferulic acid (purity > $99 \%$ ) was purchased from Sigma Aldrich, US. The commercial kits used for blood plasma and urine enzymatic assays and biochemical analysis were obtained from BioSource International Inc., US. Analytical grade chemicals were purchased from authentic suppliers.

\section{Experimental animals}

Thirty adult male Sprague-Dawley rats (180 $220 \mathrm{~g}$ ) were obtained from the laboratory animal center of Baoji People's Hospital. The animals were acclimatized to laboratory conditions at room temperature and $12 \mathrm{~h}$ light $/ 12 \mathrm{~h}$ dark cycle, and were permitted ad libitum access to standard diet and drinking water prior to the experiment. Ethical approval for this study was obtained from the Ethical Committee of Baoji People's Hospital (Medical Branch) with approval no. 20170801001. All animals were maintained according to the recommendations of National Institutes of Health (IRAC1985, Appendix B)[14] and the ethical committee.

\section{Experimental design}

The rats were assigned to five groups (six rats per group): group I (normal control), group II (urolithiatic control), groups III and IV (experimental urolithiatic groups), and Group V (positive control urolithiasis). Urolithiasis was induced by administration of ethylene glycol $(0.75 \% \mathrm{v} / \mathrm{v})$ in drinking water to all animal groups (except for group I) for 28 consecutive days. Rats in groups III and IV were orally administered ferulic acid at doses of 40 and $80 \mathrm{mg} / \mathrm{kg}$, respectively for 28 days, using gavage, while rats in group were orally administered the standard anti-urolithiatic drug cystone $(750 \mathrm{mg} / \mathrm{kg})$ for 28 days, via gavage [15]. After 28 days, the animals were placed in individual metabolic cages so to collect urine samples for analysis. The rats were thereafter anaesthetized and blood samples were taken from the retro-orbital axis, after which they were sacrificed through cervical dislocation. The kidneys were excised for use in biochemical and histopathological analyses. The blood samples were centrifuged at $2000 \mathrm{~g}$ for $10 \mathrm{~min}$, and the plasma samples were collected. A section of the kidney was fixed in $10 \%$ formalin for histopathological evaluation. The remaining kidney tissues were homogenized in $50 \mathrm{mM}$ phosphate buffer $(\mathrm{pH} 7.4)$ and centrifuged at $11000 \mathrm{~g}$ for $15 \mathrm{~min}$ at $4^{\circ} \mathrm{C}$ to obtain supernatants for enzymatic assays. All samples were placed in a refrigerator at $-80^{\circ} \mathrm{C}$ prior to analysis.

\section{Biochemical assays}

The levels of kidney damage markers i.e. aspartate aminotransferase (AST), alkaline phosphatase (ALP), gamma-glutamyl transferase (GGT), alanine aminotransferase (ALT), and lactate dehydrogenase $(\mathrm{LDH})$ in serum and 
kidney were estimated using commercial autoanalyzer kits (Shimadzu CL-7200, Japan). The levels of urolithiasis stone and renal markers calcium oxalate, magnesium, uric acid, inorganic phosphorus, urea, and creatinine in serum, urine and kidney were assayed using commercial kit autoanalyzer (Shimadzu CL-7200, Japan). Kidney levels of antioxidant enzymes catalase (CAT), glutathione peroxidase (GPx), superoxide dismutase (SOD) and renal oxidative stress markers reduced glutathione (GSH), and lipid peroxidation (LPO) were analyzed with commercial kits (Sigma Diagnostic kit, USA).

\section{Histopathological examination}

Kidney sections fixed in $10 \%$ formalin were processed and stained ( $H$ \& E). The slides were studied at $\times 100$ magnification under microscope. The kidney tissue sections were scored for renal calculi formation under polarized optical microscope in 10 different fields. The scores were given according to the number of stones formed at multiple sites: $0=$ no crystal formed, 1 $=$ less than 10 crystals formed, 2 = less than 30 crystals formed, $3=$ less than 50 crystals formed, $4=$ less than 75 crystals formed, and $5=$ more than 75 crystals formed.

\section{Statistical analysis}

Data are presented as mean \pm standard error of mean (SD) for six individual experiments. Oneway analysis of variance (ANOVA) was applied for statistical analysis, followed by Tukey's multiple comparison test. All statistical analyses were carried out using SPSS software (version 19.0, Chicago, USA). Values of $p<0.05$ were considered significant.

\section{RESULTS}

\section{Effect of ferulic acid on general variables in rats}

The effect of ferulic acid on general variables such as change in body weight, urine $\mathrm{pH}$, urine volume, water consumption, dry weight and wet weight of kidney were measured after 28 days of the treatment. The results obtained are displayed in Table 1. There were significant changes $(p<$ 0.05 ) in all the general variables in rats in group II which showed decreases in body weight and urine volume, when compared to normal control rats. The changes in urine $\mathrm{pH}$, water consumption, and weights of dry and wet kidney of Group II rats indicated the presence of kidney stone, when compared to rats of in group I. Ferulic acid-treated rats of groups III and IV showed significantly improved levels of the variables in contrast to group II rats $(p<0.05)$. A higher dose of ferulic acid $(80 \mathrm{mg} / \mathrm{kg})$ produced better changes in all the variables than the lower dose. Group V rats showed significantly altered $(p<0.05)$ levels of the variables compared to group II rats.

\section{Effect of ferulic acid on serum and kidney biochemical markers of urolithiasis}

Rats in group II showed marked increases in the serum and kidney levels of all the biochemical markers, relative to rats in the normal control group, demonstrating the extent of damage caused by ethylene glycol. However, these serum and kidney biochemical markers were significantly and dose-dependently suppressed by treatment with ferulic acid $(p<0.05)$. This shows that the ethylene glycol-induced kidney damage was markedly mitigated by ferulic acid. Group V rats also showed significantly decreased levels of serum and kidney biochemical markers, when compared with those in group II. The results are displayed in Table 2.

\section{Effect of ferulic acid on kidney stone and renal markers}

As shown in Table 3, group II rats clearly showed the presence of calcium oxalate crystals, and significantly increased serum and urine levels of stone and renal markers, when compared to the normal control rats $(p<0.05)$. However, these increases were significantly and dosedependently reversed by treatment with ferulic acid, as shown in the results in groups III and IV rats which clearly had no renal calculi formation. Rats in group $\mathrm{V}$ also showed significantly lowered levels of stone and renal markers in serum and urine, when compared to group II rats

Table 1: General variables measured after 28 days of treatment

\begin{tabular}{lccccc}
\hline Variable & Group I & Group II & Group III & Group IV & Group V \\
\hline Urine $\mathrm{pH}$ & $6.4 \pm 0.01$ & $8.6 \pm 0.04^{*}$ & $7.2 \pm 0.14^{\#}$ & $6.98 \pm 0.09^{\#}$ & $6.5 \pm 0.96^{\#}$ \\
Urine volume $(\mathrm{mL} / 24 \mathrm{~h})$ & $17.32 \pm 0.21$ & $10.64 \pm 0.14^{*}$ & $15.96 \pm 0.32^{\#}$ & $16.72 \pm 0.04^{\#}$ & $18.21 \pm 0.74^{\#}$ \\
Water intake $(\mathrm{mL} / 24 \mathrm{~h})$ & $17.96 \pm 0.04$ & $18.19 \pm 0.12^{*}$ & $17.95 \pm 0.65^{\#}$ & $17.73 \pm 0.41^{\#}$ & $17.42 \pm 0.63^{\#}$ \\
Change in body weight $(\mathrm{g})$ & $5.7 \pm 0.47$ & $2.5 \pm 0.08^{*}$ & $3.49 \pm 0.06^{\#}$ & $4.64 \pm 0.12^{\#}$ & $5.12 \pm 0.46^{\#}$ \\
Wet kidney weight $(\mathrm{mg})$ & $0.63 \pm 0.29$ & $1.26 \pm 0.12^{*}$ & $0.88 \pm 0.15^{\#}$ & $0.76 \pm 0.61^{\#}$ & $0.71 \pm 0.01^{\#}$ \\
Dry kidney weight $(\mathrm{mg})$ & $0.18 \pm 0.06$ & $0.39 \pm 0.69^{*}$ & $0.28 \pm 0.63^{\#}$ & $0.21 \pm 0.03^{\#}$ & $0.16 \pm 0.18^{\#}$ \\
\hline \multicolumn{2}{l}{ Results are presented as mean $\pm \mathrm{SD}(\mathrm{n}=6) .{ }^{*} p<0.05$, in comparison to group I; \#p<0.05, relative to group II }
\end{tabular}


Table 2: Effect of ferulic acid on serum and kidney biochemical markers of urolithiasis

\begin{tabular}{llccccc}
\hline Sample & Group & ALP & AST & ALT & GGT & LDH \\
\hline \multirow{3}{*}{ Serum } & Group I & $136.74 \pm 3.27$ & $46.21 \pm 1.65$ & $36.32 \pm 1.63$ & $14.65 \pm 0.46$ & $224.21 \pm 2.54$ \\
(U/L) & Group II & $296.21 \pm 1.63^{*}$ & $147.45 \pm 5.42^{*}$ & $65.14 \pm 0.63^{*}$ & $36.41 \pm 0.27^{*}$ & $471.21 \pm 8.58^{*}$ \\
& Group III & $214.23 \pm 1.98^{\#}$ & $111.64 \pm 1.95^{\#}$ & $54.36 \pm 0.86^{\#}$ & $28.36 \pm 0.51^{\#}$ & $380.21 \pm 6.32^{\#}$ \\
& Group IV & $165.21 \pm 2.64^{\#}$ & $99.32 \pm 1.21^{\#}$ & $48.14 \pm 0.45^{\#}$ & $20.14 \pm 0.21^{\#}$ & $262.22 \pm 7.11^{\#}$ \\
& Group V & $151.23 \pm 2.13^{\#}$ & $87.36 \pm 0.93^{\#}$ & $41.23 \pm 1.40^{\#}$ & $16.63 \pm 0.32^{\#}$ & $241.36 \pm 2.41^{\#}$ \\
\hline \multirow{3}{*}{ Kidney } & Group I & $25.36 \pm 0.64$ & $4.21 \pm 0.77$ & $17.63 \pm 0.91$ & $19.36 \pm 1.02$ & $6.36 \pm 0.08$ \\
(U/mg & Group II & $63.87 \pm 0.73^{*}$ & $9.65 \pm 0.36^{*}$ & $57.56 \pm 1.53^{*}$ & $78.96 \pm 1.72^{*}$ & $17.54 \pm 0.29^{*}$ \\
protein) & Group III & $39.36 \pm 1.47^{\#}$ & $5.90 \pm 0.26^{\#}$ & $34.74 \pm 1.18^{\#}$ & $40.21 \pm 1.04^{\#}$ & $11.24 \pm 0.39^{\#}$ \\
& Group V & $31.21 \pm 1.13^{\#}$ & $5.12 \pm 0.38^{\#}$ & $24.96 \pm 0.89^{\#}$ & $28.36 \pm 0.98^{\#}$ & $9.36 \pm 0.28^{\#}$ \\
\hline Reun
\end{tabular}

Results are given as mean $\pm \mathrm{SD}(\mathrm{n}=6) .{ }^{*} p<0.05$, relative to group I; $\# p<0.05$, in comparison to group II

\section{Effect of ferulic acid on antioxidant enzymes and oxidative stress markers in kidney}

There was clear evidence of oxidative stress pattern in group II rats, with significantly reduced levels of GSH, SOD, GPx, and CAT, whereas the tissue damage marker LPO was significantly elevated, when compared to group I rats $(p<$ $0.05)$. However, ferulic acid significantly inhibited oxidative stress in groups III and IV rats by replenishing the levels of GSH, SOD, GPx, and CAT while reducing the level of LPO, when compared to rats in group II. Similar results were seen in group $\mathrm{V}$ rats, which had significant elevations in GSH, SOD, GPx, CAT and reduction in LPO, relative to group II $(p<0.05)$. These results are presented in Table 4.

\section{Effect of ferulic acid on the histopathological features}

Histopathological evaluation of the kidney sections of all the groups of rats were done to examine the changes in pathology due to the challenge of ethylene glycol and the antiurolithiatic effect of ferulic acid. The renal calculi crystals formed were scored and the results are shown in Figure 1. Renal calculi formation was not visible in the normal control rats of group I, whereas an average score of 5.5 was given for rats in group II, indicating high level of renal calculi. In contrast, crystal formation scores were significantly reduced in the groups of rats treated with ferulic acid. Group V rats also exhibited similar results i.e. significantly reduced renal calculi formations.

Table 3: Effect of ferulic acid on stone and renal markers in urine and plasma

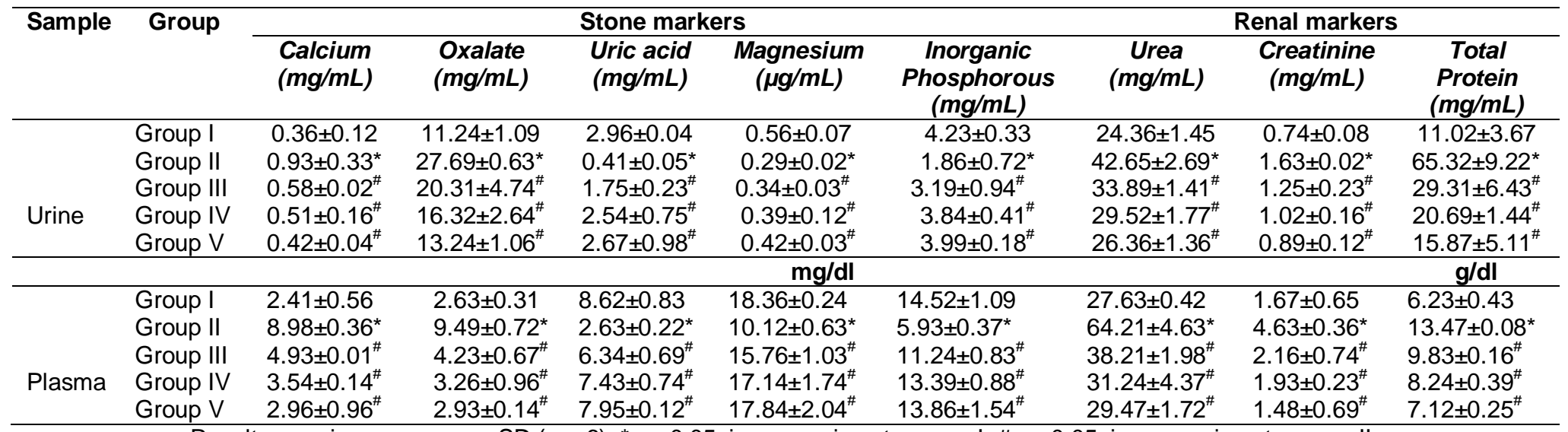

Results are given as mean \pm SD $(n=6) ;{ }^{*} p<0.05$, in comparison to group I; \#p<0.05, in comparison to group II

Table 4: Effect of ferulic acid on some antioxidant enzymes and oxidative stress markers in kidney tissues of the control and experimental rats

\begin{tabular}{lccccc}
\hline Group & $\begin{array}{c}\text { SOD (U/mg } \\
\text { protein) }\end{array}$ & $\begin{array}{c}\text { GPx (U/mg } \\
\text { protein) }\end{array}$ & $\begin{array}{c}\text { CAT (U/g } \\
\text { protein) }\end{array}$ & $\begin{array}{c}\text { GSH (U/mg } \\
\text { protein }\end{array}$ & $\begin{array}{c}\text { LPO (U/mg } \\
\text { protein) }\end{array}$ \\
\hline Group I & $1.75 \pm 0.14$ & $0.98 \pm 0.12$ & $1.32 \pm 0.12$ & $1.36 \pm 0.13$ & $0.64 \pm 0.06$ \\
Group II & $0.94 \pm 0.11^{*}$ & $0.42 \pm 0.04^{*}$ & $0.96 \pm 0.09^{*}$ & $1.02 \pm 0.10^{*}$ & $0.94 \pm 0.08^{*}$ \\
Group III & $1.24 \pm 0.13^{\#}$ & $0.61 \pm 0.04^{\#}$ & $1.12 \pm 0.10^{\#}$ & $1.12 \pm 0.11^{\#}$ & $0.82 \pm 0.05^{\#}$ \\
Group IV & $1.41 \pm 0.12^{\#}$ & $0.72 \pm 0.06^{\#}$ & $1.20 \pm 0.11^{\#}$ & $1.20 \pm 0.14^{\#}$ & $0.73 \pm 0.06^{\#}$ \\
Group V & $1.51 \pm 0.15^{\#}$ & $0.86 \pm 0.05^{\#}$ & $1.22 \pm 0.10^{\#}$ & $1.29 \pm 0.14^{\#}$ & $0.69 \pm 0.06^{\#}$ \\
\hline
\end{tabular}

Results are given as mean \pm SD $(\mathrm{n}=6) ;{ }^{*} p<0.05$, relative to group I; $\# p<0.05$, in comparison to group II 


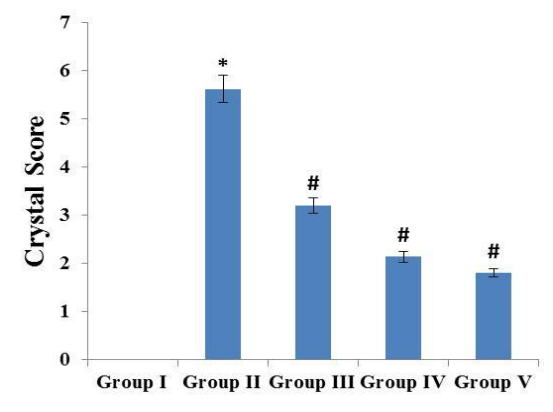

Figure 1: Effect of ferulic acid on the renal calculi formation score. Results are given as mean $\pm \mathrm{SD}(\mathrm{n}=$ 6). ${ }^{*} p<0.05$, relative to group I; $\# p<0.05$, in comparison to group II

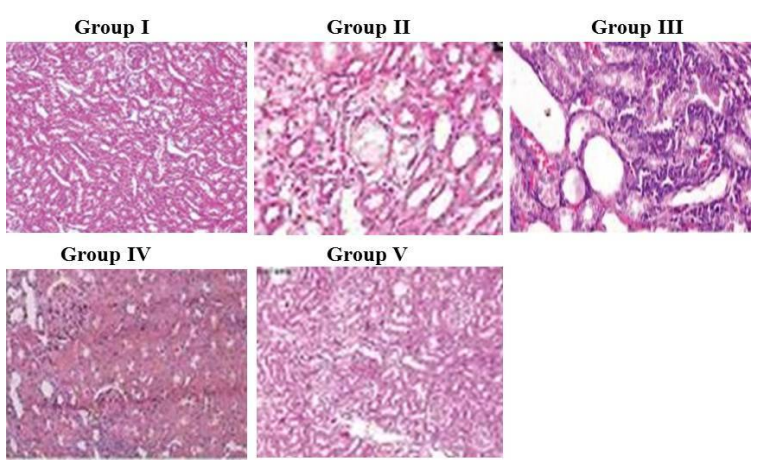

Figure 2: Histopathological alterations by ferulic acid in kidney of control and experimental rats. Group II rats had severe tissue damage as depicted by tubular necrosis, glomerular and blood vessel congestion, and enlarged renal tubules with calculi formation. In contrast, there was less damage in the ferulic acidtreated rats, and fewer renal calculi formation. $\mathrm{H}$ \& $\mathrm{E}$ staining at $100 \times$ magnification

The kidney sections of Group I rats were normal, with no abnormalities in the tissues as shown in Figure 2. Group II rats had severe damage to the tissues as revealed by tubular necrosis, glomerular and blood vessel congestion, enlarged renal tubules with calculi formation induced by ethylene glycol. In contrast, ferulic acid treatment significantly prevented ethylene glycol-induced damage in a dose-dependent manner, as shown in groups III and IV rats which had reduced renal calculi crystals. Group V rats exhibited minor damage to the tissues, when compared to the urolithiasis control rats of group II.

\section{DISCUSSION}

Research has shown that antioxidants from plants act as potential anti-urolithiatic agents for solving the problem of kidney stone formation and its recurrence after surgery [16]. In this study, the anti-urolithiatic potential of ferulic acid was tested against ethylene glycol-induced renal calculi formation in rats. Rat model of ethylene glycol-induced urolithiasis results in formation of renal calculi consisting calcium oxalate crystals [17]. It was found from the results that ferulic acid was able to inhibit the renal calculi formation effectively through regulation of antioxidant enzymes against oxidative stress induced by ethylene glycol. Being a phenolic antioxidant, ferulic acid was able to scavenge free radicals formed during the biotransformation of ethylene glycol hence the level of LPO was reduced and GSH was preserved.

It has been reported previously that plant-derived antioxidants ameliorate changes in urine and serum calcium, magnesium, oxalate, urine $\mathrm{pH}$, uric acid, urea, and creatinine of urolithiasis models. This is consistent with the results showing that ferulic acid was able to prevent the formation of renal calculi by altering these general variables [18]. Urolithiasis is also caused by inflammation of kidney due to the toxic effects of toxicants [19]. Ferulic acid prevented the inflammatory process through its antiinflammatory properties, thereby reducing the possibilities of renal calculi formation. The levels of serum, urine and kidney markers of stone formation and renal damage clearly showed renal calculi formation in the urolithiasis control group. Aspartate transaminase (AST) and ALT are located within the cellular cytoplasm and are also identified as markers of damage to the cellular integrity of kidney [20]. Other enzymes like ALP, GGT, and LDH are also elevated in the serum and urine due to damage of the cellular structure in kidney.

Oxidative stress is a major cause of cellular structural damage which leads to the release of these enzymes into the blood and urine [21]. The kidney tissues in group II rats were severely damaged by ethylene glycol-induced oxidative stress. These results are also supported by the histopathological findings which revealed kidney damage at multiple sites. Renal calculi are often formed in oxidative stress-induced damage to kidney tissues caused by oxidative stress. The antioxidant enzymes SOD, GPX, CAT and GSH regulate free radical formation in the internal environment [22]. The activities of these antioxidant enzymes were markedly reduced in the urolithiasis rats due to a collapse of the antioxidant defense system, leading to oxidative stress and renal calculi formation. The antioxidant and anti-inflammatory properties of ferulic acid are attributed to its ability to suppress oxidative stress and prevent renal calculi formation as shown in the results. Ferulic acid was able to protect the kidney tissues and preserve the antioxidant enzymes which regulate ROS, thereby exerting an anti-urolithiatic effect. 
The anti-urolithiatic potential of ferulic acid in ethylene glycol-induced renal calculi formation was supported by the biochemical and histopathological findings.

\section{CONCLUSION}

The anti-urolithiatic effect of ferulic acid is attributed to its antioxidant nature which prevents oxidative stress induced by ethylene glycol, thus preventing renal calculi formation. The prevention of oxidative stress and the use of natural antioxidants are potential agents for the prevention of kidney stone formation. Therefore, ferulic acid is a potential drug candidate with strong pharmacological effect against kidney stone-related ailments. However, there is need for further studies to elucidate the detailed mechanism involved in the mitigation of urolithiasis by ferulic acid.

The gastrointestinal polyps and associated symptoms disappeared after approximately 1 year of TCHM therapy without any complications during the follow-up. This case suggests that TCHM could play an important role in the treatment of gastrointestinal polyps. It may be a better choice for the patients who refuse surgery or cannot be surgically operated on, because the Chinese traditional medicine treatment of chronic gastritis and gastrointestinal polyps method is simple and less painful. Relevant data are however limited, and randomized controlled trials are still needed to confirm its efficacy in a larger population.

\section{DECLARATIONS}

\section{Acknowledgement}

The authors would like to thank Baoji Health and Family Planning Bureau Scientific Research Project (no. 2015-38, 2016-28) for financial support.

\section{Conflict of Interest}

No conflict of interest associated with this work.

\section{Contribution of Authors}

The authors declare that this work was done by the authors named in this article and all liabilities pertaining to claims relating to the content of this article will be borne by them.

\section{REFERENCES}

1. Edvardsson VO, Indridason OS, Haraldsson G, Kjartansson O, Palsson R. Temporal trends in the incidence of kidney stone disease. Kidney Int 2013; 83(1): 146-152.

2. Zhou J, Jin J, Li X, Zhao Z, Zhang L, Wang Q, Li J, Zhang $Q$, Xiang $S$. Total flavonoids of Desmodium styracifolium attenuates the formation of hydroxyl-1proline-induced calcium oxalate urolithiasis in rats. Urolithiasis 2018; 46(3): 231-241.

3. Vanachayangkul $P$, Byer $K$, Khan S, Butterweck V. An aqueous extract of Ammi visnaga fruits and its constituents khellin and visnagin prevent cell damage caused by oxalate in renal epithelial cells. Phytomed 2010; 17(8-9): 653-658.

4. Mi J, Duan J, Zhang J, Lu J, Wang H, Wang Z. Evaluation of antiurolithic effect and the possible mechanisms of Desmodium styracifolium and Pyrrosiae petiolosa in rats. Urol Res 2012; 40(2): 151-161.

5. Khan SR. Reactive oxygen species as the molecular modulators of calcium oxalate kidney stone formation: evidence from clinical and experimental investigations. $J$ Urol 2013; 189(3): 803-811.

6. Saremi J, Kargar-Jahroomi H, Poorahmadi M. Effect of Malva neglecta Wallr on ethylene glycol induced kidney stones. Urol J 2015; 12(6):2387-2390.

7. Gnanaraj C, Shah MD, Tan TS, lqbal $M$. Hepatoprotective mechanism of Lygodium microphyllum (Cav.) R.Br. through ultrastructural signaling prevention against carbon tetrachloride (CC/4)-mediated oxidative stress. Biomed. Pharmacother 2017; 92: 1010-1022.

8. Arulselvan P, Wen CC, Lan CW, Chen YH, Wei WC, Yang NS. Dietary administration of scallion extract effectively inhibits colorectal tumor growth: Cellular and molecular mechanisms in mice. PLoS One 2012; 7(9):e44658.

9. Gnanaraj C, Shah MD, Makki JS, lqbal M. Hepatoprotective effects of Flagellaria indica $L$. are mediated through the suppression of pro-inflammatory cytokines and oxidative stress markers in rats. Pharm Biol 2016; 54(8): 1420-1433.

10. Patel PK, Patel MA, Vyas BA, Shah DR, Gandhi TR. Antiurolithiatic activity of saponin rich fraction from the fruits of Solanum xanthocarpum Schrad \& Wendl (Solanaceae) against ethylene glycol induced urolithiasis in rats. J Ethnopharmacol 2012; 144: 160170.

11. Khan A, Khan SR, Gilani AH. Studies on the in vitro and in vivo antiurolithic activity of Holarrhena antidysenterica. Urol Res 2012; 40(6): 671-681.

12. Mir SM, Ravuri HG, Pradhan RK, Narra S, Kumar JM, Kuncha M, Kanjilal S, Sistla R. Ferulic acid protects lipopolysaccharide-induced acute kidney injury by suppressing inflammatory events and upregulating antioxidant defenses in Balb/c mice. Biomed Pharmacother 2018; 100: 304-315. 
13. Srinivasan M, Sudheer AR, Menon, VP. Ferulic acid: Therapeutic potential through its antioxidant property. $J$ Clin Biochem Nut 2007; 40:92-100.

14. National Research Council (US) Committee for the Update of the Guide for the Care and Use of Laboratory Animals. Guide for the Care and Use of Laboratory Animals. 8th edition. Washington (DC): National Academies Press (US); 2011. Key Concepts. Available from: https://www.ncbi.nlm.nih.gov/books/NBK54054/

15. Mitra SK, Gopumadhavan S, Vengkataranganna MV, Sundaram R. Effect of cystone, a herbal formulation, on glycolic acid-induced urolithiasis in rats. Phytotherapy Res 1998; 12: 372-374.

16. Xiang S, Zhou J, Gan S, Rong XL, Li J, Wang S. Antiurolithiatic activity of aqueous extract from Desmodium styracifolium (Osb.) Merr. on renal calcium oxalate in rats. Chin J Exp Surg 2013; 30(6): 1166

17. Ghale-Salimi MY, Eidi M, Ghaemi N, Khavari-Nejad RA. Antiurolithiatic effect of the taraxasterol on ethylene glycol induced kidney calculi in male rats. Urolithiasis 2018; 46(5): 419-428.

18. Yuruk E, Tuken M, Sahin C, Kaptanagasi AO, Basak K, Aykan S, Muslumanoglu AY, Sarica K. The protective effects of an herbal agent tutukon on ethylene glycol and zinc disk induced urolithiasis model in a rat model. Urolithiasis 2016; 44(6): 501-507.

19. Aggarwal D, Gautam D, Sharma M, Singla SK. Bergenin attenuates renal injury by reversing mitochondrial dysfunction in ethylene glycol induced hyperoxaluric rat model. Eur J Pharmacol 2016; 791: 611-621.

20. Zuo J, Khan A, Glenton PA, Khan SR. Effect of NADPH oxidase inhibition on the expression of kidney injury molecule and calcium oxalate crystal deposition in hydroxy-L-proline-induced hyperoxaluria in the male Sprague-Dawley rats. Nephrol Dial Transplant 2011; 26: 1785-1796.

21. Hsu CC, Lien JC, Chang CW, Chang CH, Kuo SC, Huang TF. Yuwen02f1 suppresses LPS-induced endotoxemia and adjuvant-induced arthritis primarily through blockade of ROS formation, NF-kB and MAPK activation. Biochemical Pharmacol 2013; 85: 385-395.

22. Mulay SR, Kulkarni OP, Rupanagudi KV, Migliorini $A$, Darisipudi MN, Vilaysane A, Muruve D, Shi $Y$, Munro F, Liapis H, Anders HJ. Calcium oxalate crystals induce renal inflammation by NLRP3-mediated IL-1beta secretion. J Clin Invest 2013; 123: 236-246. 